\title{
ADVANCED ALTITUDE SIMULATION FACILITY P8 - CURRENT STATUS
}

\author{
C. Pauly, D. Suslov, and O. J. Haidn \\ Institute of Space Propulsion \\ Deutsches Zentrum für Luft- und Raumfahrt(DLR) \\ Lampoldshausen 74239, Germany
}

\begin{abstract}
The paper reports the current status of a DLR Lampoldshausen project towards the design, erection, and operation of an advanced altitude simulation facility at the European R\&T Facility P8. The system will allow for testing subscale thrust chamber assemblies (TCAs) including surrounding supersonic flow around the nozzle. This facility will allow for investigation into the specific features of altitude simulation facilities but also on the interaction of nozzle and its exhaust plume and the surrounding coflow for subsonic, transitional, and low supersonic coflow conditions. The design bases entirely on the broad experience on design and operation of various altitude simulation facilities such as the satellite engine bench P1.0, the cryogenic and storable upper-stage engine facilities P4.1 and P4.2, sophisticated engineering design tools and continuous numerical effort. Knowledge about nozzle and thrust chamber design and operation bases on broad investigations carried out at the cold-flow facility P6.2 and the hot-fire M3 and P8 test benches.
\end{abstract}

\section{INTRODUCTION}

The investigation of rocket engine behavior under real flight conditions requires the solution of several complex physical problems. During the ascent of the launcher the ambient conditions vary drastically. While the temperature decrease has only a minor influence, the change of the surrounding flow from subsonic to supersonic and vanishing of atmospheric pressure cause severe variations on the boundary conditions for engine operation. Launchers like the European ARIANE 5, the American Space Shuttle, or the Japanese H2 are designed in such a way that their core engines are ignited at ambient pressure on the launching pad

This is an Open Access article distributed under the terms of the Creative Commons Attribution-Noncommercial License 3.0, which permits unrestricted use, distribution, and reproduction in any noncommercial medium, provided the original work is properly cited. 
and operate for several hundred seconds with decreasing ambient pressure [1]. For the VULCAIN 2 engine of the ARIANE 5 launcher, the engine shuts down at an altitude of about $215 \mathrm{~km}$, where the pressure is lower than 6-10 mbar and surrounding flow velocities have reached values around $7000 \mathrm{~m} / \mathrm{s}$.

Obviously, these variations in boundary and operating conditions have an impact on engine design and performance. Flow structures resulting from the launcher afterbody may impact the nozzle and the arising asymmetric loads may deform the nozzle structure or couple with the flow inside the nozzle causing changes in the internal nozzle flow which can finally result in flow separation and side loads [2-5]. In order to clarify the impact of these phenomena on engine operation in sufficient detail, different facilities are available at each scale level and DLR operates a variety of such facilities. Engine facility P1.0 is designed for satellite propulsion systems in the thrust range of 400-600 N. Engine facility P6.2 is a facility designed for cold-flow subscale studies with a geometric scale of 1:25 taking VULCAIN 2 engine as a reference; P4.1 $(180 \mathrm{kN})$ and P4.2 (35 kN) are full-scale systems designed for altitude simulation testing of upper-stage engines such as VINCI and AESTUS [7, 6, 8]. Until now, there is no facility available in Europe which allows for an altitude simulation on engines of VULCAIN 2 class. Only during the VULCAIN 2 recovery program, P5 bench was modified in setting up a Load Simulation Device (LSD) in order to establish low-pressure conditions $(\sim 200$ mbar $)$ around the engine [9].

The planned Advanced Altitude Simulation (AAS) at P8 test bench, a facility which allows for 1:8 subscale testing (VULCAIN 2 class), aims at filling the gap between full-scale upper-stage facilities P4.1 and P4.2 and cold-flow facility P6.2 [10]. It is designed to allow for altitude simulation of subscale TCAs with coflow around the engine. The current design limits the thrust level to roughly $30 \mathrm{kN}$ and a supersonic surrounding flow to a Mach number of 2. In the end, the AAS P8 test bench will be designed to allow for:

- investigations into the coupling of internal and external flow phenomena under hot-gas conditions and subscale level to improve the identification and quantitative description of the dominating physical phenomena; among these phenomena are nozzle deformation, occurrence of abnormal side loads, buffeting, postcombustion in areas where hydrogen-rich exhaust gases mix with surrounding air;

- investigation into the design and operation of specific components of such altitude simulation facilities to enable a more detailed understanding of their operation and/or to build a basis for future developments; of particular interest are diffusers, ejectors, pressure development, cooling methods, and flow phenomena during transient operation of the engine; 
- application and development of optical diagnostic methods to investigate the flow phenomenon in the region of interest, of particular interest are classical methods such as Schlieren, high-speed imaging in the visual and ultraviolet (UV) range or more sophisticated techniques like BackwardOriented Schlieren (BOS) [11], infrared (IR) thermometry, or nonintrusive velocity measurement tools;

- model development and validation for engineering analytical and numerical computational fluid dynamics (CFD) tools applied for simulation-based predictions of specific components and their interaction during steady-state and transient operation phases and for prediction of flow interactions; and

- R\&D into the coupling between specific features of TCAs and such facilities.

Obviously, the final design will be a compromise between the specific requirements of these different goals. However, they certainly have requirements in common, well-known and controllable boundary conditions, and a controlled variation of operating conditions.

\section{DESIGN METHODOLOGY AND APPROACH}

The design for such a sophisticated facility requires verified tools for the different components and subsystems and their interactions. Design, erection and operation of P4.1 facility provided a large experimental and numerical data base and finally yielded a set of verified engineering design tools which can be regarded as the state-of-the-art in the field $[12,13]$. However, the operating conditions at P8 and those foreseen for AAS P8 are quite different. One the one hand, while P8 facility allows for higher combustion chamber pressures which may exceed $25 \mathrm{MPa}$, the AAS P8 should allow for coflow around chamber and nozzle with the pressure level of the coflow adjustable to ascent conditions. On the other hand, the geometrical size of P8 is much smaller and requires a down-scaling of the major components for altitude simulation.

Additionally to the experience gathered during the P4.1 project, the behavior of nozzles at varying external pressure and flow conditions have been studied in detail at cold-flow facility P6.2. This experimental effort has been accompanied by detailed numerical studies and, obviously, the results obtained there provide a broad data base for nozzle flows, coupling phenomena between nozzle and outer coflow. Furthermore, during these research activities, various optical diagnostic techniques have been applied and the combination of all these data, experiences, and lessons learned will be taken into account for AAS P8 design [14-17]. 


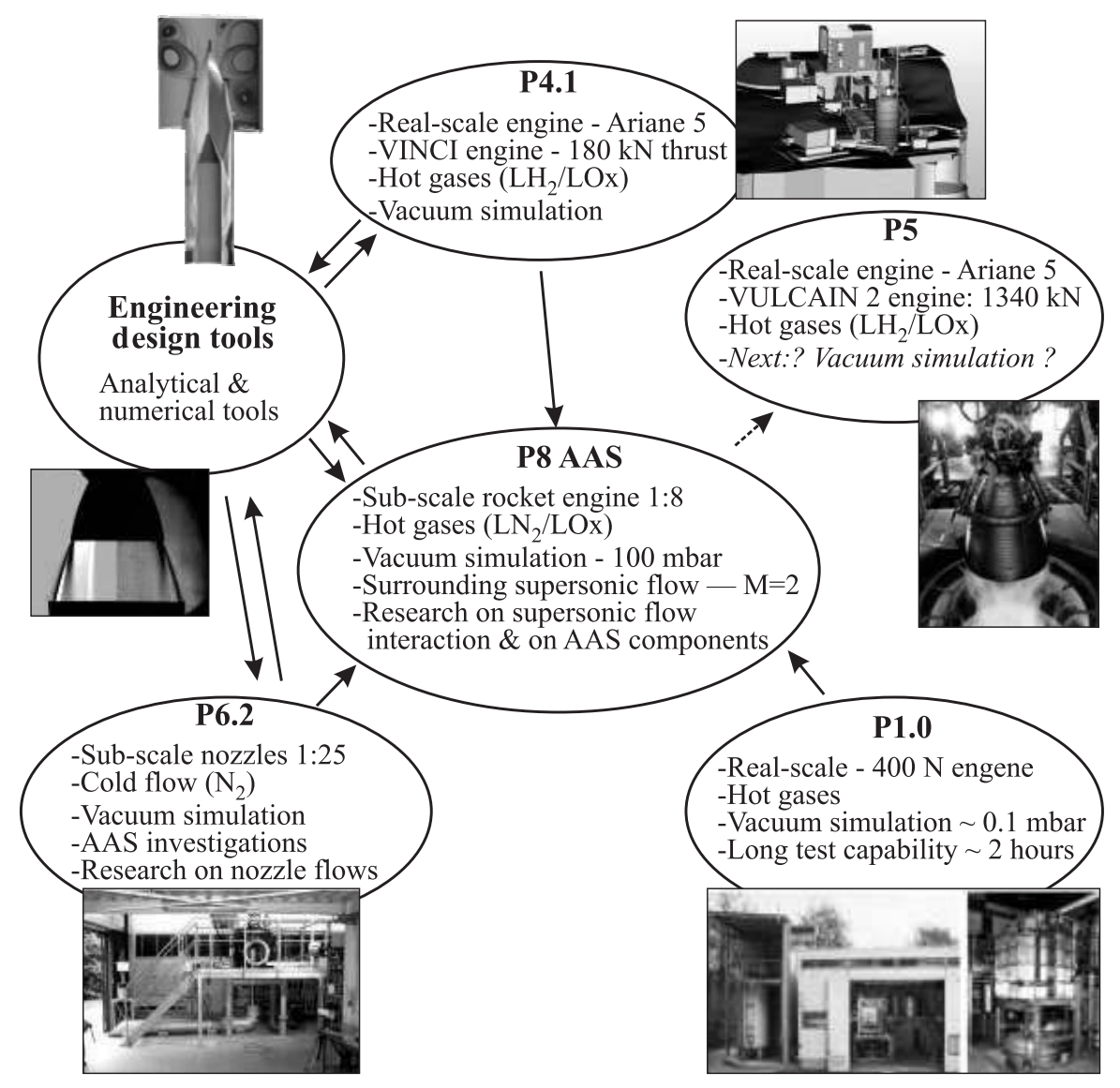

Figure 1 Illustration of AAS P8 design methodology

The third element of the design process are the operational experience, the experimental data base, and the knowledge about test facility and test specimen interaction gathered within the last decade at P8. The different users at P8 performed test campaigns in a broad range of operating conditions of combustion chamber pressure (30 to 250 bar), mass flow rates (from 0.5 to $17 \mathrm{~kg} / \mathrm{s}$ ), and propellants $\left(\mathrm{GH}_{2}, \mathrm{LH}_{2}, \mathrm{GCH}_{4}, \mathrm{LOx}\right)$ for main chamber, gas generator, and staged combustion conditions [18, 19]. Figure 1 summarizes the design methodology. A combination of all the assets mentioned previously in a thoroughly followed design process will result in a successful AAS P8. 


\section{COLD FLOW ACTIVITIES}

\subsection{Advanced Altitude Simulation Setup at P6.2}

The P6.2 facility is used for research into flow separation and side load phenomena of thrust nozzles having different contours in cold-flow conditions. It allows for two experimental setups: one where the nozzle flow exists into ambientpressure environment and another where the nozzle operates in a vacuum chamber (VC) which can be operated in a mode with continuous adaptation of the ambient pressure to an actual flight phase.

The test bench is equipped with gaseous nitrogen feeding system that allows working with a maximal pressure of 55 bar and with maximal mass flow of around $4 \mathrm{~kg} / \mathrm{s}$.

Figure 2 shows a picture of P6.2 facility in the altitude simulation mode, setup with diffuser and exhaust section connected without the upstream system of propelling and bleed gas supply. The test facility capabilities are summarized in Table 1, for more detailed information see [6].

The AAS setup is composed of several subcomponents and a schematic of the specific setup is shown in Fig. 3. All features which are ASS specific such as the surrounding nozzle for secondary flow, the glass cylinder to allow for optical access, and the ejector system with secondary throat diffuser and nozzle are marked with dashes.

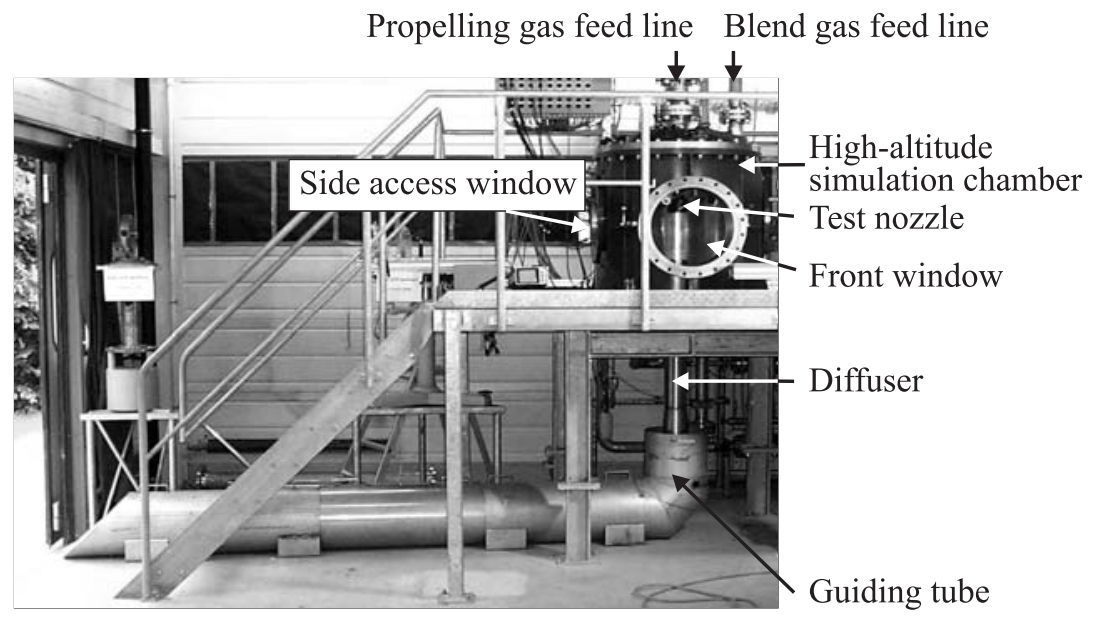

Figure 2 Main features of P6.2 altitude simulation test facility 
Table 1 The P6.2 test facility capabilities

\begin{tabular}{ll}
\hline \multicolumn{1}{c}{ Subcomponent } & \multicolumn{1}{c}{ Description } \\
\hline TCA & Cold nitrogen gas (ambient total temperature); maxi- \\
Subscale chamber + nozzle & mal feeding pressure: 55 bar; maximal mass flow rate: \\
& $\sim 4 \mathrm{~kg} / \mathrm{s}$ \\
\hline Altitude Simulation & Vacuum pressure $<100 \mathrm{mbar}$; minimal VC pressure \\
System (ASS) & $\sim 8 \mathrm{mbar}$ \\
Vacuum chamber + super- & Vacuum chamber dimensions: $800 \mathrm{~mm}$ in diameter and \\
sonic diffuser & $1000 \mathrm{~mm}$ high \\
& Three optical access windows: $2 \times 400 \mathrm{~mm}$ and \\
& $1 \times 500 \mathrm{~mm}$ diameters; Interchangeability of diffuser \\
& types: cylinder, secondary throat and centerbody \\
\hline Surrounding Flow & Atmospheric air $($ ambient total temperature and pres- \\
System (SFS) & sure) \\
Surrounding flow nozzle & Mass flow rate: from 0.3 to $\sim 1 \mathrm{~kg} / \mathrm{s}$ \\
+ ejector & Feeding pressure ejector nozzle: maximum 45 bar \\
& Mass flow rate: $\sim 3 \mathrm{~kg} / \mathrm{s}$ \\
& Suction behavior of the ejector: $0.3 \mathrm{~kg} / \mathrm{s}$ for 0.1 bar \\
\hline
\end{tabular}

\subsubsection{Subsystems and components description}

TCA: Obviously, one of the main components of the setup is the thrust chamber with its dominating part for altitude purposes, the nozzle. Since P6.2 is a cold-flow facility, the device mounted into P6.2 resembles only features such as chamber pressure, contraction, and expansion nozzle contour. Figure 3 shows that the setup allows for optical access to the nozzle and the area of intersection of the plume with the surrounding high-speed gas. Both classical bell and dual bell nozzles having different nozzle contours are the objects of investigations using the AAS setup. Since the first object of interest is a downscaled VULCAIN 2 type chamber, all the experiments were performed with a thrust-optimized bell type nozzle with similar expansion ratio.

ASS: The ASS consists of the VC and the central supersonic diffuser which provides for the achievement of vacuum pressure inside the $\mathrm{VC}$ and the recompression of the supersonic flow exhausting from the nozzle. However, in order to reach sufficient low pressures (some millibars) inside the $\mathrm{VC}$ and to ensure that full flowing nozzle conditions are achieved, the diffuser has to be in a chocked condition or, that is to say, to be "started," meaning that at least in one section of the diffuser, supersonic flow conditions have to be established. For the AAS test campaign, the second throat diffuser is used which provides for a VC pressure smaller than 50 mbar. 


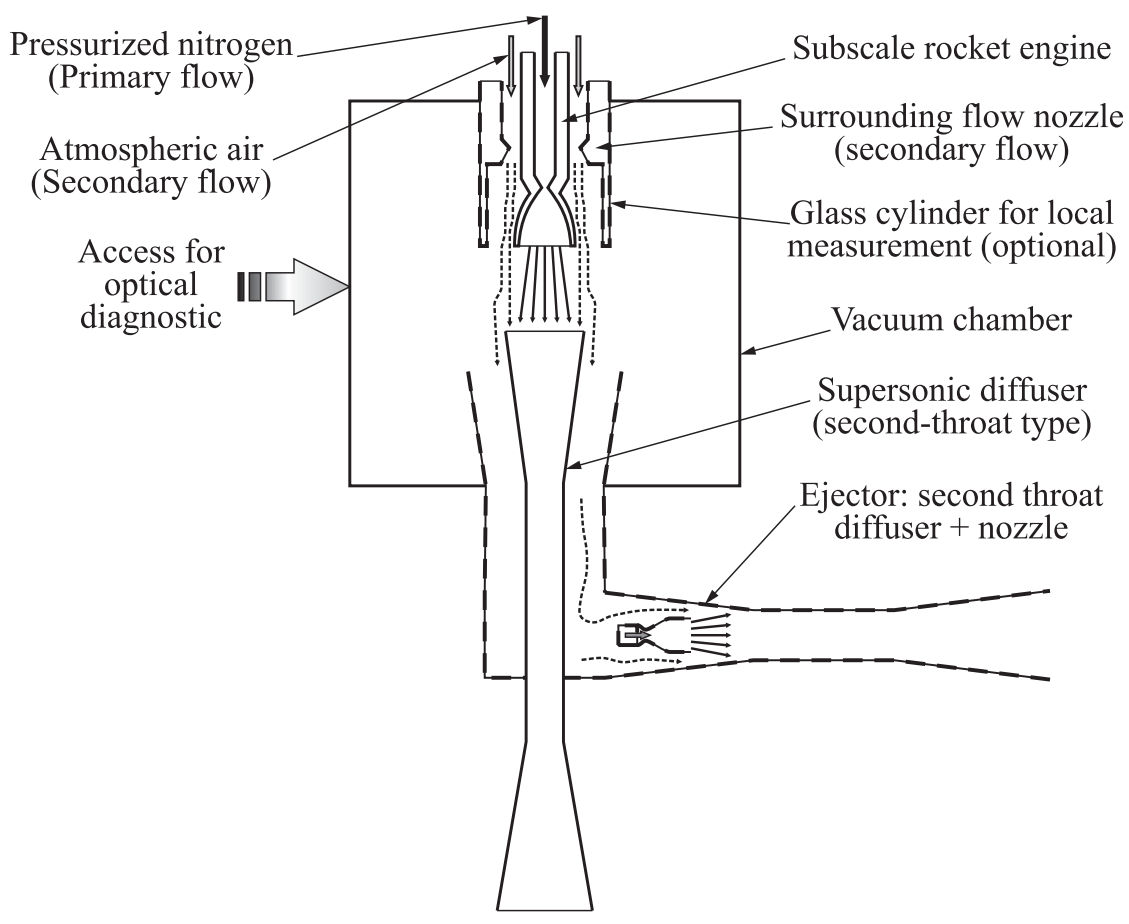

Figure 3 Schematic of the AAS setup at P6.2

SFS: The components of the system which provide for the surrounding flow and its mastering within the ASS are supply lines, guiding flow paths, the nozzle for the surrounding flow, and the ejector. Additionally, the system is equipped with a glass cylinder to allow for the optical access for flow visualization and diagnostic purposes. These components establish a supersonic flow around the nozzle and allow for the aspiration of the injected secondary mass flow introduced through the surrounding flow nozzle in order to keep low pressure levels inside the VC.

\subsubsection{Optical diagnostics and measurement systems}

In order to provide for the necessary data for comparison with numerical simulations, facility and components and subsystems of interest are equipped with several pressure and temperature sensors at specific positions. The measurement system is composed of around 80 canals: $\sim 64$ x-times low-frequency canals (up to $1 \mathrm{kHz}$ ) and $\sim 16 \mathrm{x}$ (times) high-frequency canals (up to $50 \mathrm{kHz}$ ). Various optical techniques have been applied so far during different test campaigns 
at P6.2 $[4,6,14]$. For the validation campaigns reported here, the focus lays on a Z-type Schlieren method. Additionally, a new technique, Background Oriented Schlieren (BOS), which catches the optical displacement of a pattern on a structure behind the flow caused by density gradients in the flow, will be tested during the validation phase [11]. Although this method yields results comparable to a conventional Schlieren setup with a somewhat poorer resolution, it has the advantage of an easier application.

\subsection{Operation Conditions and Sample Test Results}

As of today, the majority of the tests planned for P6.2, single component and sub-systems performance as well as their interaction and coupling have already been performed. Currently, these data are evaluated and compared with numerical simulations in order to modify the engineering design tools. The tests are performed with a parabolic VULCAIN 2 like contour nozzle with the some expansion ratio. The chamber pressure is fixed to 48 bar while the vacuum pressure around the engine was kept around 30 mbar. Tests were performed with and without secondary surrounding flow. No ejector is used; the vacuum is sustained by the subscale engine - supersonic diffuser combination. The tests are performed without glass cylinder and with a conical nozzle for the secondary flow. Table 2 summarizes the operating conditions of these tests.

Figure 4 shows three Schlieren images taken with different setups (Figs. $4 a$ and $4 b$ ) at different pressure ratios. While the images of Figs. $4 a$ and $4 c$ are taken with a vertical Schlieren setup and visualize flow conditions without surrounding coflow, the image of Fig. 4b, taken with a horizontal Schlieren setup, shows the flow at a similar pressure ratio as that in Fig $4 a$ but with coflow. The remaining

Table 2 The AAS P6.2 test conditions

\begin{tabular}{cl}
\hline Tests & \multicolumn{1}{c}{ Description } \\
\hline TCA characteristics & TOP Parabola nozzle - VULCAIN 2 like contour - \\
& Throat diameter $=11 \mathrm{~mm}$ \\
& Nozzle expansion ratio $\varepsilon=53$ (optional 100) \\
& Nozzle end diameter $80 \mathrm{~mm}$ \\
& $P_{c c} \sim 48$ bar \\
& Mass flow $=1.1 \mathrm{~kg} / \mathrm{s}$ \\
\hline ASS & Vacuum pressure $\sim 30 \mathrm{mbar}$ \\
& Vacuum pressure with secondary flow $\sim 80 \mathrm{mbar}$ \\
& Distance from nozzle end to diffuser inlet around $58 \mathrm{~mm}$ \\
\hline SFS & Atmospheric air $($ ambient total temperature and pressure) \\
& Mass flow rate: $0.17 \mathrm{~kg} / \mathrm{s}$ \\
\hline
\end{tabular}




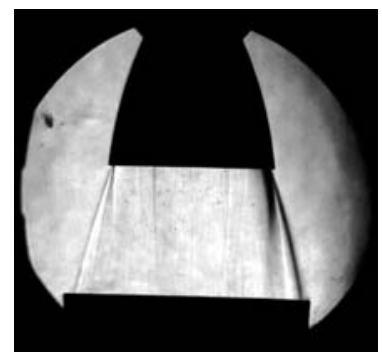

(a)

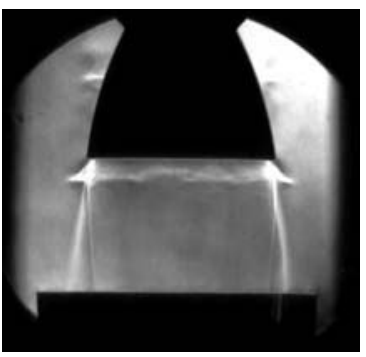

(b)

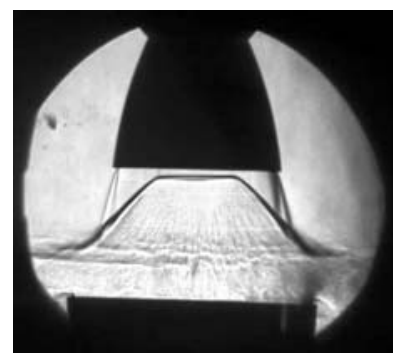

(c)

Figure 4 Schlieren images with AAS installation at P6.2: steady state without (a) and with $(b)$ secondary flow; and transient shutdown without secondary flow $(c)$

test campaigns will focus on the application of optical access installations, the BOS technique, and ejector behavior.

\section{COMPARISON OF NUMERICAL AND EXPERIMENTAL COLD-FLOW RESULTS}

\subsection{Description of the Computational Fluid Dynamics Model}

ANSYS CFX 11.0 has been used to compute the different flow situations and to compare the solutions with experimental data. Mesh generations were performed applying ANSYS ICEM 11.0. Based on symmetry assumptions, the computational effort were reduced drastically. Furthermore, only the dominating features of the system such as nozzles and diffusers were modeled completely. The VC was modeled only partially, ejector and feed lines were not taken into account. Two different geometries have been built with two-dimensional (2D) axisymmetrical domains. The mesh was built with hexahedron cells, because they provided

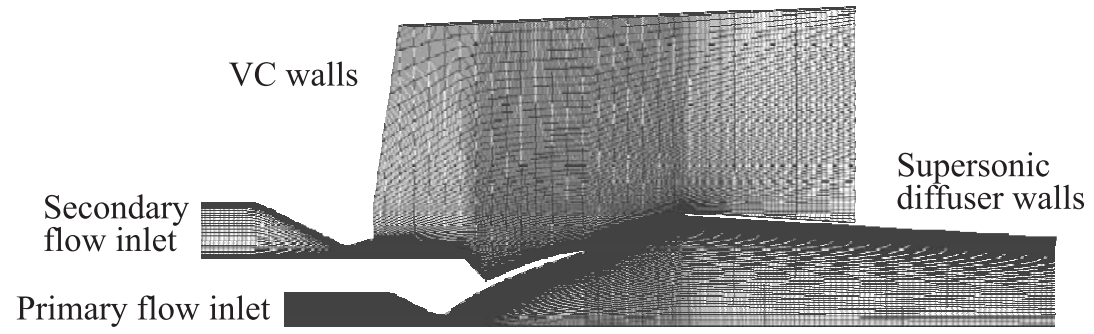

Figure 5 Computational mesh for the simulation with secondary flow 
the better mesh quality. The first mesh for the simulation without coflow was of around 76000 cells, and the second was built with 123000 cells. An additional mesh with around 300000 nodes has been built to verify that results were not dependent of the mesh size (geometry with coflow). The example of the mesh built is presented in Fig. 5. The chosen turbulence model was the shear stress transport (SST) Menter model.

Nitrogen was used to model the primary flow and air for the secondary flow. The gases were modeled with applying a perfect gas approach because there was no significant temperature gradient influencing the heat capacity. The walls were modeled as adiabatic walls. The inlets were defined through the total pressure and total temperature conditions with the corresponding measured values; like the outlet modeling.

\subsection{Comparison of Experimental and Numerical Results}

Figures $6 a$ and $6 b$ show a comparison of the experimental and numerical determined Schlieren images for the two different operating points defined in Table 2: a TCA pressure of 48 bar and a coflow mass flow rate of $179 \mathrm{~g} / \mathrm{s}$. Each of the figures is subdivided in a numerical (left) and an experimental half (right).

Figure $6 a$ compares the flow between nozzle exit and diffuser inlet for the case without secondary flow. While free jet boundary and expansion fan are clearly visible in both images, the internal shock, a well-known structure of thrustoptimized contour nozzles, is much more pronounced in the numerical Schlieren

Density gradient $Y$ (contour 3 ) $/ \mathrm{kg} / \mathrm{m}^{4}$
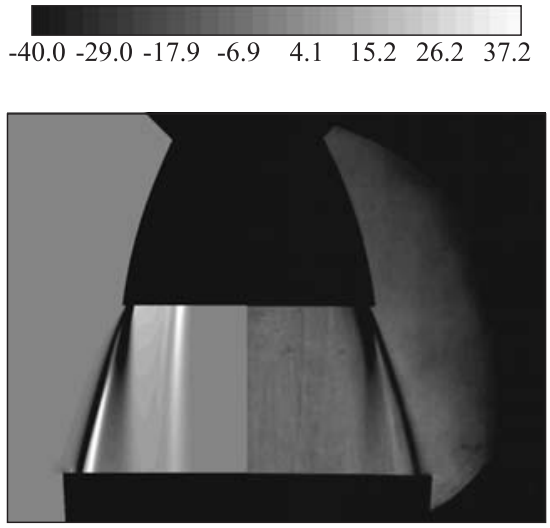

(a)
Density gradient $Z$ (contour 1 ) $/ \mathrm{kg} / \mathrm{m}^{4}$
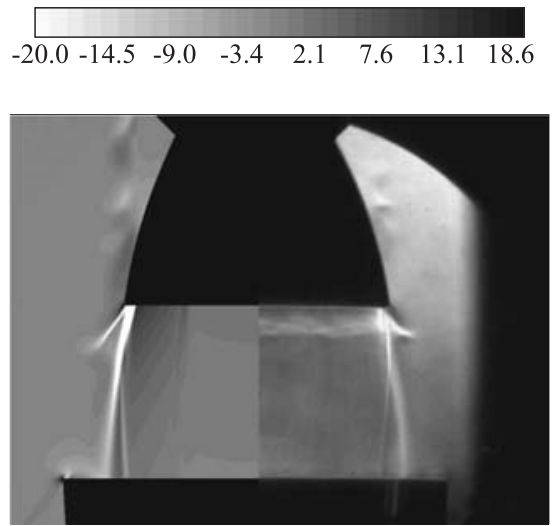

(b)

Figure 6 Comparison between experiment and simulation without $(a)$ and with $(b)$ coflowing gas 


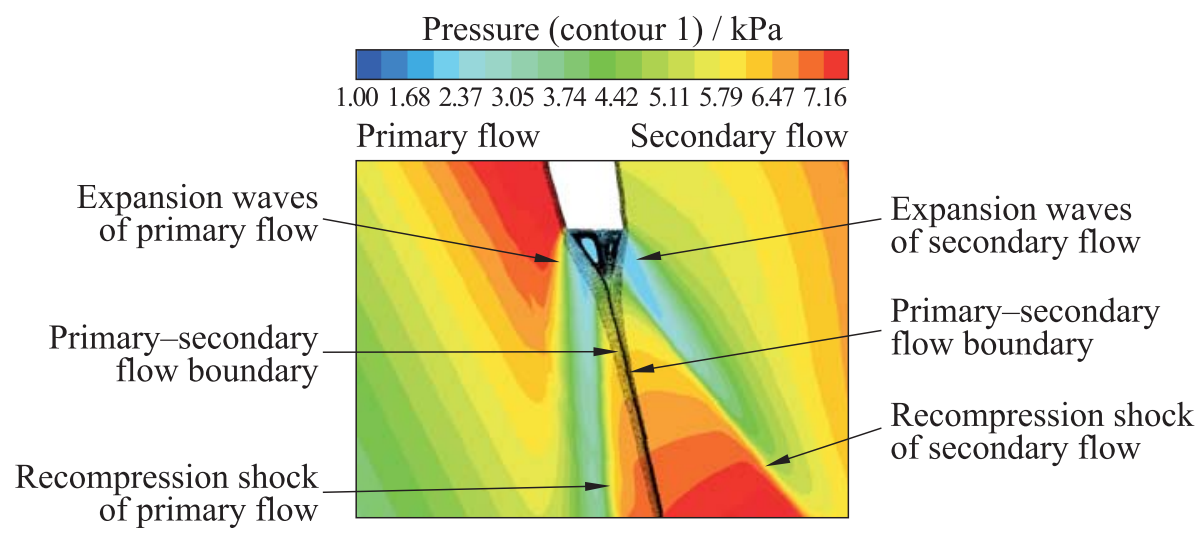

Figure 7 Exploded view of the flow configuration at the nozzle exit: CFD simulation with secondary flow pressure contours and streamlines. (Refer Pauly et al., p. 275.)

image than in the experimental one which is due to the fact that the latter is a line-of sight image. Schlieren orientation and finite camera resolution play a role as well.

Figure $6 b$ compares the same flow structure but now for the case with coflowing gas and this secondary flow is injected with Mach number of around 2 through the annular nozzle. This secondary flow surrounding the nozzle is visible on the Schlieren images in the form of small spots near the external nozzle wall. A recompression shock, resulting from the interaction of the secondary flow with the primary flow is clearly visible on both the numerical and the experimental Schlieren pictures and forms an oblique shock $\left(\sim 45^{\circ}\right.$ to the nozzle end-plane) from the nozzle end-wall lip. This shock structure is slightly smaller in experimental images than in the results of CFD and seems to be a more curved at its extremity. This shock structure is three-dimensional but axisymmetrical and appears all around the nozzle end. Because of superposition effects, the "annular shock" yields marks onto the experimental Schlieren at the end of the nozzle which seems to be inside the subscale core flow, but is, of course, not. The boundary of the primary supersonic flow is also visible in both images: it is supposed to be the limit between both jets too. Finally, the third characteristic structure is observed, it is the recompression shock going from the nozzle end into the diffuser inside the primary flow which exits from the nozzle.

Figure 7 shows a zoom of the numerical results (pressure contours) obtained with the finer mesh (about two times finer in the nozzle end - diffuser inlet region) for the case with secondary flow. The results do not show significant differences to the ones obtained with the nominal mesh both in terms of shock structure observed in the Schlieren images or in terms of VC pressure. However, interpretation of these data and consideration of theory allow for a better un- 
derstanding of the flow phenomenon at the nozzle end [18]. At both sides of the nozzle lip, supersonic jets are flowing along the walls (internal wall = primary flow; external wall $=$ secondary flow) up to the end. The abrupt change there results in expansion wave fans at the wall corners yielding local low-pressure areas on both sides downstream of the corners. These expansion waves start to overlap further downstream and form two oblique shocks, which are oriented in other directions; that is to say towards primary core flow for the primary shock and towards VC walls for the secondary shock. Behind both shocks, the pressure suddenly increases. Between both oblique shocks, the boundary between the primary and secondary flow can also be distinguished through the streamlines.

\section{FORESEEN CAPABILITY OF THE ADVANCED ALTITUDE SIMULATION SYSTEM AT P8}

The foreseen capabilities of the next AAS installation at P8 Test Facility are summarized in Table 3. While the first application clearly aims at altitude simulation applications similar to the VULCAIN 2 during launch, the facility should

Table 3 The AAS P8 test facility capabilities

\begin{tabular}{cll}
\hline Subcomponent & \multicolumn{1}{c}{ AAS P8 } & AAS P8 Vulcain 2 \\
\hline TCA & Thrust optimized parabola (TOP) & TOP nozzle \\
& Nozzle area ratio $\varepsilon=57$ (optional 100) & Area ratio $\varepsilon=57$ \\
& Nozzle exit diameter $\sim 250 \mathrm{~mm}$ & $P_{\mathrm{cc}}=115 \mathrm{bar}$ \\
& Throat diameter $=33 \mathrm{~mm}$ & Mass flow $=320 \mathrm{~kg} / \mathrm{s}$ \\
& $75<P_{\mathrm{cc}}<15 \mathrm{bar}($ nominal $85 \mathrm{bar})$ & $R_{\mathrm{of}}=7.2$ \\
& Mass flow $\sim 3.1 \mathrm{~kg} / \mathrm{s}(6 \mathrm{~kg} / \mathrm{s}$ maximum) & \\
& $5.5<R_{\text {of }}<7.5($ nominal 6.5$)$ & \\
\hline AAS & Surrounding pressure adjustable between & \\
& 1 bar and 100 mbar & \\
& Windows for optical diagnostics: Schlieren, \\
& BOS & \\
& Exchangeable primary and secondary noz- \\
& zles and supersonic diffusers & \\
& Supersonic diffuser to nozzle end distance & \\
& can be varied & \\
\hline SFS & Air or nitrogen study postcombustion of & \\
& hydrogen-rich exhaust gases & \\
& Surrounding flow up to M $=2$ & \\
& Mass flow rate up to $7 \mathrm{~kg} / \mathrm{s}$ &
\end{tabular}


as well be capable of providing the subscale testing of the majority of engines currently under discussion within the European Future Launchers Preparatory Programme (FLPP). Hence, AAS P8 will be able to contain high-thrust gasgenerator thrust chambers as well as staged combustion arrangements. Furthermore, it will be possible to use $\mathrm{LOx} / \mathrm{CH}_{4}$ instead of $\mathrm{LH}_{2} / \mathrm{LOx}$. The AAS will allow for various thrust nozzle types (TOP, truncated ideal contour (TIC), dual bell, etc.) up to an expansion ratio of $\varepsilon=100$. Additional to conventional measurement techniques, AAS will allow for extensive flow visualization techniques (high-speed Schlieren and BOS).

\section{HOT GASES ACTIVITIES}

For providing experimental data similar to those obtained at P6.2, nozzle experiments have to be performed at P8 already in the early phase of the project. These experiments have to be accompanied by appropriate numerical studies to finally have sufficient data available for the preliminary design review (PDR) scheduled for later this year.

\subsection{Subscale Rocket Engine for the Advanced Altitude Simulation at P8}

As the first step, subscale hot-gas tests were performed without altitude simulation to obtain data on the behavior of the $\mathrm{P} 8$ test bench and gain experience about nozzle specific measuring techniques which will be applied later. For these investigations, a VULCAIN 2-type subscale nozzle extension has been developed. Manufactured of Inconell ${ }^{\circledR} 600$, the nozzle consists of two parts: the first one (from $\varepsilon=5$ to 32 ) possesses cooling channels operating with ambient-temperature hydrogen and the second one (from $\varepsilon=32$ up to 57) uses the hydrogen coming out of the cooling channels of the first part for film cooling. Figure 8 shows the subscale TCA prior to testing at P8.

The design combines the classical milling

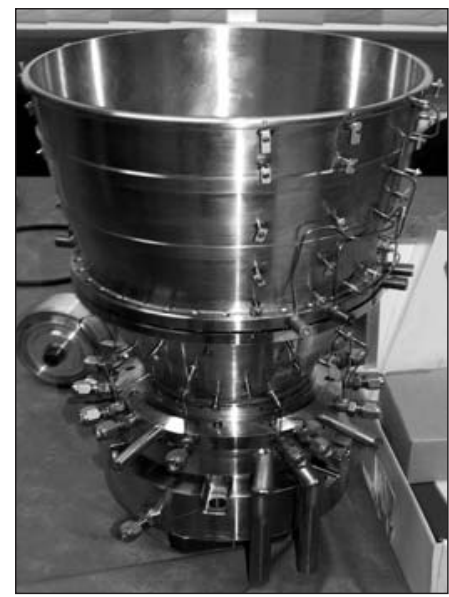

Figure 8 Subscale TCA for P8 technique for machining the cooling channel into the liner with electroplating of the outer shell (Nickel) and thus avoids welding of the closeout. This approach minimizes the buildup of strain during 


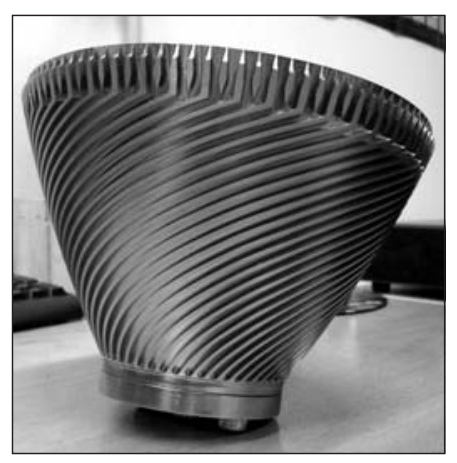

Figure 9 Helical cooling channels of the first part of the nozzle fabrication and testing. The nozzle skirt (second part) is exchangeable and allows for investigations into different nozzle concepts, i.e., dual-bell contour nozzles.

The cooling channels of the first part are helical (Fig. 9). The coolant supply is split into four different parts which are equipped with closed loop control valves to allow for an individual adjustment of the cooling flow rates of the four quadrants. In a standard setup, the hydrogen coolant enters the nozzle to form a supersonic film and the variable slot geometry allows for the simulation of different injection velocities by constant mass flow ratios between the main flow and coolant film. Figure 10 shows the TCA with the nozzle sections mounted at P8.

The test campaign reported here has been performed for typical engine sea level start-up conditions and included a variation of the combustion chamber pressure and mixture ratio later into the test between 50 bar up to 130 bar and oxygen-to-fuel ratio from 6 up to 7.3, respectively.

The instrumentation of the test specimen allows for rather detailed investigations of flow separation induced side loads through an arrangement of load cells implemented into the interface between the chamber and nozzle which are

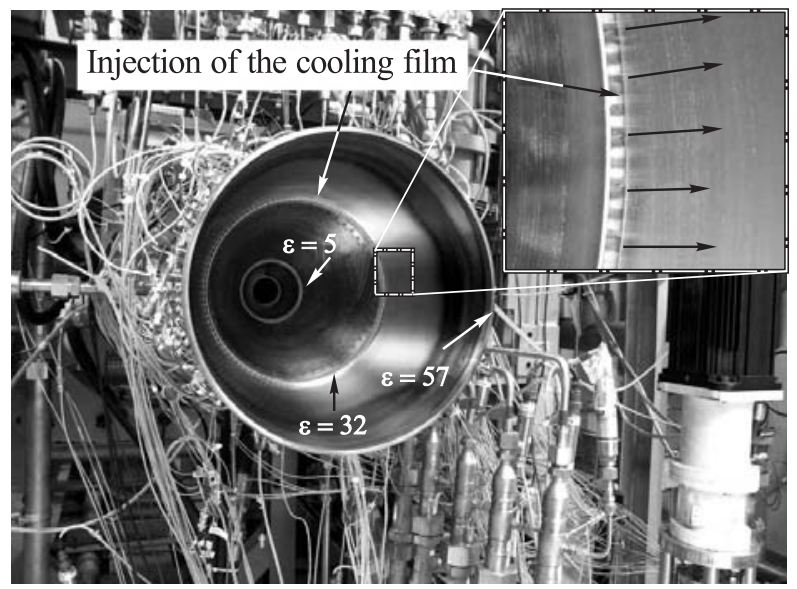

Figure 10 View of TCA mounted a P8 


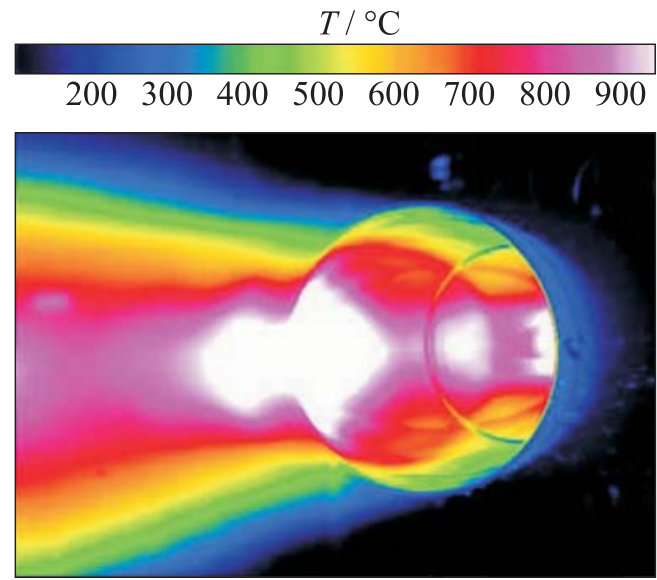

(a)

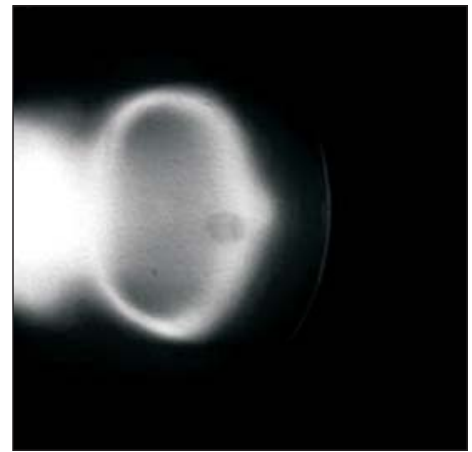

(b)

Figure 11 Plume radiation images: IR $(a)$ and UV $(\mathrm{OH})(b)$. (Refer Pauly et al., p. 279.)

not reported here. Conventional pressure and temperature sensors have been combined with cameras detecting in the IR and UV spectra (Fig. 11). Both the colored IR and the black-and-white $\mathrm{OH}$ images quite nicely reveal some key features of the flow. A more detailed look at the exhaust jet structure during the transient start-up between 3.68 and 5.28 s, shown subsequently in Figs. $12 a-12 d$, which relate to a nozzle pressure ratio (NPR $=p_{c} / p_{0}$ ) change from 37 to 43 , reveals that although film cooling is already established to some extend, the flow in the nozzle undergoes the process of restricted shock separation (RSS) to free shock separation (FSS) transition well-known for this type of nozzle contours. Although combustion chamber pressure and resulting NPR increase, as well as the injected mass flow rate of film cooling, flow separation in Fig. $12 c$ is clearly visible much nearer to the throat than in Fig. $12 b$.

Furthermore, a more detailed look at these pictures gives the impression that the diameter of the exhaust jet inside the nozzle directly after separation is larger for the lower NPR (see Fig. 12b) than for the higher NPR (see Fig. 12c), the effect typical for RSS-FSS transition.

\subsection{Preliminary Design Studies of Advanced Altitude Simulation Facility P8}

The installation was designed using internal numerical and analytical tools. These tools have been validated and upgraded based on the experimental re- 


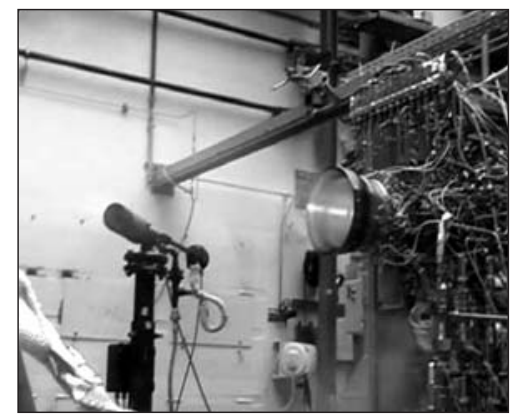

(a)

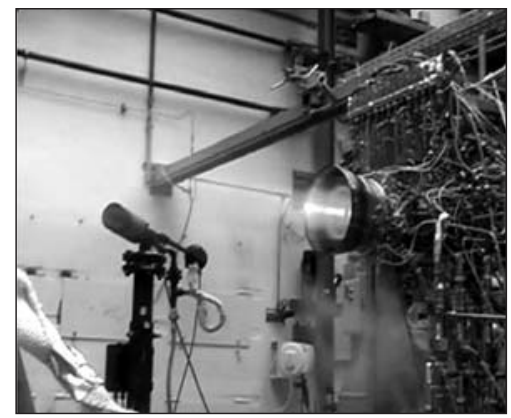

(c)

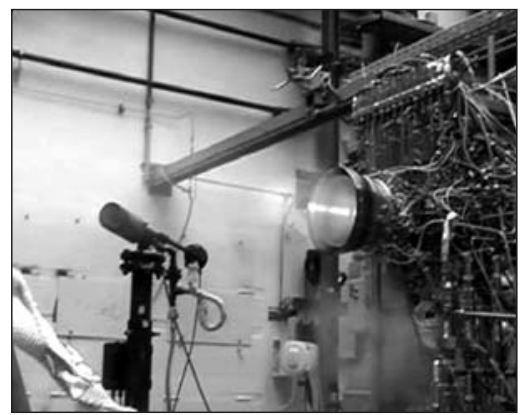

(b)

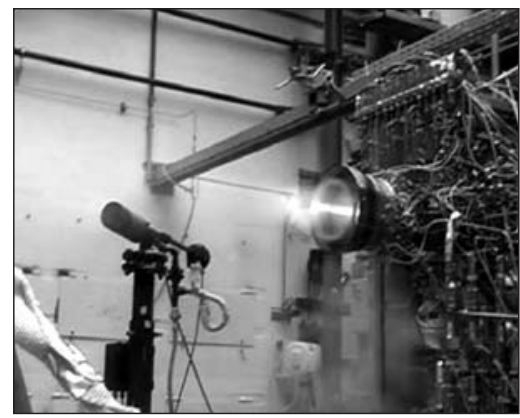

(d)

Figure 12 Flow visualization of nozzle exhaust jet during transient start-up for nozzle pressure ratios NPR ranging from 37 to 43: (a) $t=3680 \mathrm{~ms}$; $(b) 4280$; (c) 4320; and $(d) t=5280 \mathrm{~ms}$

sults obtained at different test facilities in DLR Lampoldshausen: in particular, at P6.2, P8, and P4.1 test benches. For example, CFD simulations were performed in order to verify the feasibility of the concept and to optimize the design. For the very preliminary design of the installation, 2D axisymmetric simulations were performed.

Figure $13 a$ shows the mesh used for these preliminary design studies. The simulations account for the TCA, the surrounding and central supersonic diffuser, and the annular secondary-flow diffuser. Different geometries were computed such as different secondary-flow nozzle geometries (conical or plug type nozzles), secondary-flow annular diffuser sizes, or with different gas models (with and without afterburning).

The Mach number contours for three different CFD simulations are shown in Fig. 13b. From bottom to top, the Mach number contours are presented for increasing outer diameter of the annular diffuser. Below a certain cross-sectional 


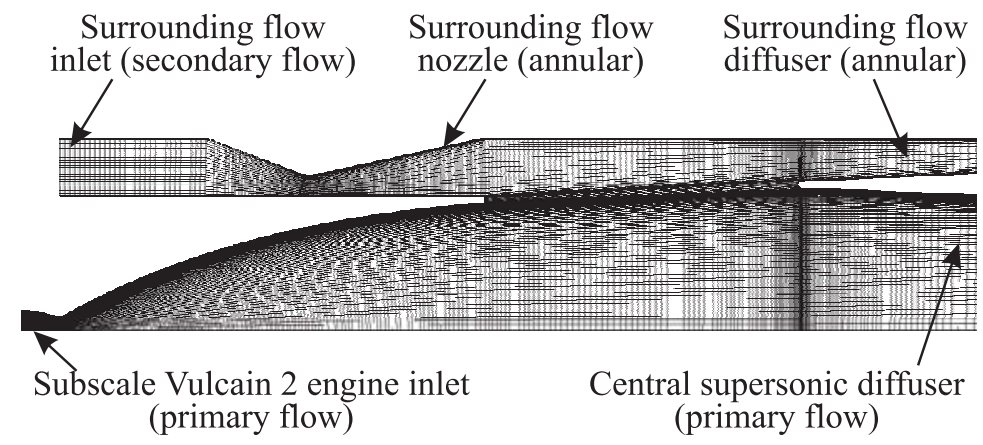

(a)

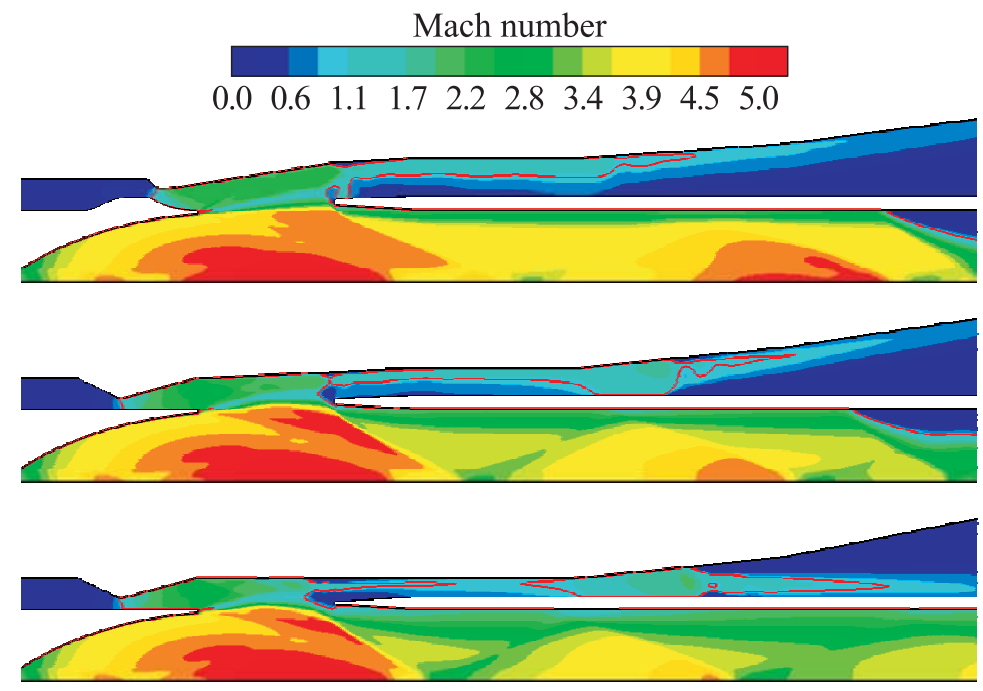

(b)

Figure 13 Illustration of the preliminary design study of the AAS installation at P8: (a) mesh for CFD simulations; and (b) CFD results (Mach number contours). (Refer Pauly et al., p. 281.)

area (bottom), the flow along the annular diffuser reaches supersonic velocities and in this case, the "annular diffuser throat" is chocked and pressures upstream this region are higher than in nonchocked condition. This phenomenon has to be avoided in order to keep low pressures in regions where supersonic flows interact, i. e., from the nozzle exit to the diffuser inlet. 
The major goals of this preliminary design study were:

- measurements of the mass flow rate in the annular secondary diffuser, that is to say, the mass flow which has to be aspired by the ejector system;

- determination of gas temperature in the annular secondary diffuser which is essential for dimensioning of a cooling system upstream of the ejector;

- determination of thermal loads at all surfaces of the components exposed to hot gases in order to improve the cooling system design; and

- evaluation of optical access and flow structures in order to optimize optical diagnostics.

\section{SUMMARY AND CONCLUDING REMARKS}

Based on broad experimental and numerical experience with design, erection and operation of altitude simulation facilities, and test benches for investigations of nozzle flow phenomena, DLR is currently gathering experimental data in specially designed experiments at different test facilities accompanied by detailed numerical studies to fill (still existing) knowledge gaps with the final aim to design and erect an altitude simulation test position at the P8 facility. Up to date, cold-flow studies of different altitude simulation setups at P6.2 have been performed accompanied by appropriate numerical analyses of these different flow situations applying ANSYS CFX. In parallel to these P6.2 related activities, a hot-gas subscale TCA which includes a TOP contoured nozzle with film cooling similar to VULCAIN 2 has been designed and tested at the P8 test facility. Furthermore, a first set of preliminary numerical studies of an advanced altitude simulation setup at P8 has been performed as well. All these data will be used to prepare the preliminary design review scheduled for later this year.

The AAS P8 will allow for the operation of subscale TCAs with coflowing gas up to $\mathrm{M} \sim 2$ at vacuum pressure around 100 mbar. Finally, this facility will allow for studies into the coupling of the hot plume from different nozzle contours with the surrounding flow, into the nozzle flow separation, resulting in side loads during varying ambient pressure and flow conditions as well as into the investigation of the specific altitude simulation subsystems, complex optical diagnostics, and the verification of numerical tools.

\section{ACKNOWLEDGMENTS}

The authors would like to acknowledge the contributions of their colleagues K. Schäfer, H. Zimmermann, C. Böhm, and M. Fricke for their valuable input. 


\section{REFERENCES}

1. Isakovic, S. J., J. Hopkins, and J. P. Hopkins. 2004. International reference guide to space launch systems. 4th ed. AIAA.

2. Nave, L. H., and G. A. Coffey. 1972. Sea level side loads in high-area-ratio rocket engines. AIAA Paper No. 73-1284.

3. Frey, M., R. Rýden, Th. Alziary de Roquefort, G. Hagemann, P. James, T. Kachler, P. Reijasse, R. Schwane, and R. Stark. 2002. European cooperation on flow separation control. 4th Conference (International) on Launcher Technology "Space Launcher Liquid Propulsion. "Liege, Belgium.

4. Stark, R., O. J. Haidn, C. Böhm, and H. Zimmermann. 2005. Cold flow testing of dual bell nozzles in altitude simulation chambers. 1st European Conference on Aerospace Sciences (EUCASS) Proceedings. Moscow.

5. Verma, S. B., R. Stark, and O. J. Haidn. 2006. Relation of shock unsteadiness towards the origin of side-loads inside a thrust optimized parabolic rocket nozzle. Aerospace Sci. Technol. 10(6):474-83.

6. Kronmüller,H., K. Schäfer, H. Zimmermann, and R. Stark. 2002. Cold gas subscale test facility P6.2 at DLR Lampoldshausen. 6th Symposium (International) on Propulsion for Space Transportation of the XXI Century Liquid Space Propulsion.

7. Schäfer, K., and H. Zimmermann. 2004. Simulation of flight conditions for rocket engine qualification. 2nd Conference (International) on Green Propellants for Space Propulsion. Cagliari, Italy. ESA SP-557.

8. Schäfer, K., and H. Zimmermann. 2006. Development and operational conditions of VINCI Altitude simulation test bench P4.1. AIAA Paper No. 2006-4902.

9. Haberzettl, A., D. Gundel, H. Zimmermann, E. Poulin, J. F. Delange, Y. Prevot, and M. Kestemann. 2005. VULCAIN 2 flight load simulation device (LSD). 1st European Conference on Aerospace Sciences (EUCASS) Proceedings. Moscow.

10. Koschel W. W., and O. J. Haidn. 1998. P8 - the new French/German test facility for $\mathrm{H}_{2} / \mathrm{O}_{2}$ high pressure combustion research. Int. J. Hydrogen Energy 23(8):68394.

11. Richard, H., and M. Raffel. 2001. Principle and applications of the background oriented Schlieren (BOS) method. Measurement Sci. Technol. 12:1576-85.

12. Verma, S. B., and O.J. Haidn. 2005. Goertler vortex formation during shutdown sequence inside a thrust optimized parabolic (TOP) rocket nozzle. AIAA Paper No. 2005-518.

13. Schäfer, K., H. Zimmermann, and C. Pauly. 2008. Operational conditions of P4.1 altitude simulation for VINCI upper stage engine. AIAA Paper No. 2008-4840.

14. Stark, R., H. Kronmüller, D. Zerjeski, and B. Wagner. 2003. Advanced flow simulation techniques in cold gas subscale nozzles, a comparison. AIAA Paper No. 20035180.

15. Preuss, A., D. Preclik, C. Mäding, J. Görgen, S. Soller, O. J. Haidn, M. Oschwald, R. Clauss, R. Arnold, and J. Sender. 2008. LOx/methane technology efforts for future liquid rocket engines. Space Propulsion 2008. Heraklion, Greece. 
16. Lux, J., and O. J. Haidn. 2009. Flame stabilization in high pressure LOx/ $\mathrm{CH}_{4}$ rocket engine combustion. J. Propul. Power 25(1):15-23.

17. Arnold, R., D. Suslov, and O. J. Haidn. 2009. Film cooling of accelerated flow in subscale combustion chamber. J. Propul. Power 25(2):443-51.

18. Korst, H. H., and W. L. Chow. 1959. Research on transonic and supersonic flow of a real fluid at abrupt increases in cross section. Mechanical Engineering Report.

19. Appolloni, L., P. Baiocco, Y. Prel, and P. Supie. 2008. Launchers technological demonstrator status. Hamamatsu, Japan. ISTS-2008-g-28. 